\title{
Differentiation and localization of interneurons in the developing spinal cord depends on DOT1L expression
}

\section{CURRENT STATUS: UNDER REVISION \\ - Molecular Brain \$BMC}

Angelica Gray de Cristoforis

Albert-Ludwigs-Universitat Freiburg

Francesco Ferrari

Max-Planck-Institut fur Immunbiologie und Epigenetik

Frédéric Clotman

Universite Catholique de Louvain

Tanja Vogel

v tanja.vogel@anat.uni-freiburg.deCorresponding Author

ORCiD: https://orcid.org/0000-0002-0602-9133

DOI:

$10.21203 / \mathrm{rs} .2 .23363 / \mathrm{v} 1$

\section{SUBJECT AREAS}

Cellular \& Molecular Neuroscience

\section{KEYWORDS}

Spinal Cord, Interneuron, Specification, Localization, Methyltransferase, DOT1L, Epigenetics 


\section{Abstract}

Genetic and epigenetic factors contribute to the development of the spinal cord. Failure in correct exertion of the developmental programs, including neurulation, neural tube closure and neurogenesis of the diverse spinal cord neuronal subtypes results in clinical phenotypes with variable severity. The histone methyltransferase Disruptor of Telomeric 1 Like (DOT1L), which mediates histone H3 lysine 79 (H3K79) methylation, is fundamental for proper development of the cerebral cortex and cerebellum, and here we report on its essential role for development of the spinal cord. Conditional inactivation of DOT1L using Wnt1-cre as driver in the developing murine spinal cord did not result in neural tube closure defect (NTCD). Transcriptome analysis revealed that DOT1L deficiency favored differentiation over progenitor proliferation. Dot1l -CKO mainly decreased the numbers of dII interneurons expressing Lhx2 . Loss of DOT1L affected localization but not generation of dI2, dI3, and dI5 interneurons. The resulting derailed interneuron patterns might be responsible for increased cell death that occurred at the late developmental stage E18.5. Together our data indicate that DOT1L is essential for subtype- specific neurogenesis, migration and localization of interneurons in the developing spinal cord, in part by regulating transcriptional activation of Lhx2 .

\section{Full Text}

Due to technical limitations, full-text HTML conversion of this manuscript could not be completed. However, the manuscript can be downloaded and accessed as a PDF.

\section{Figures}



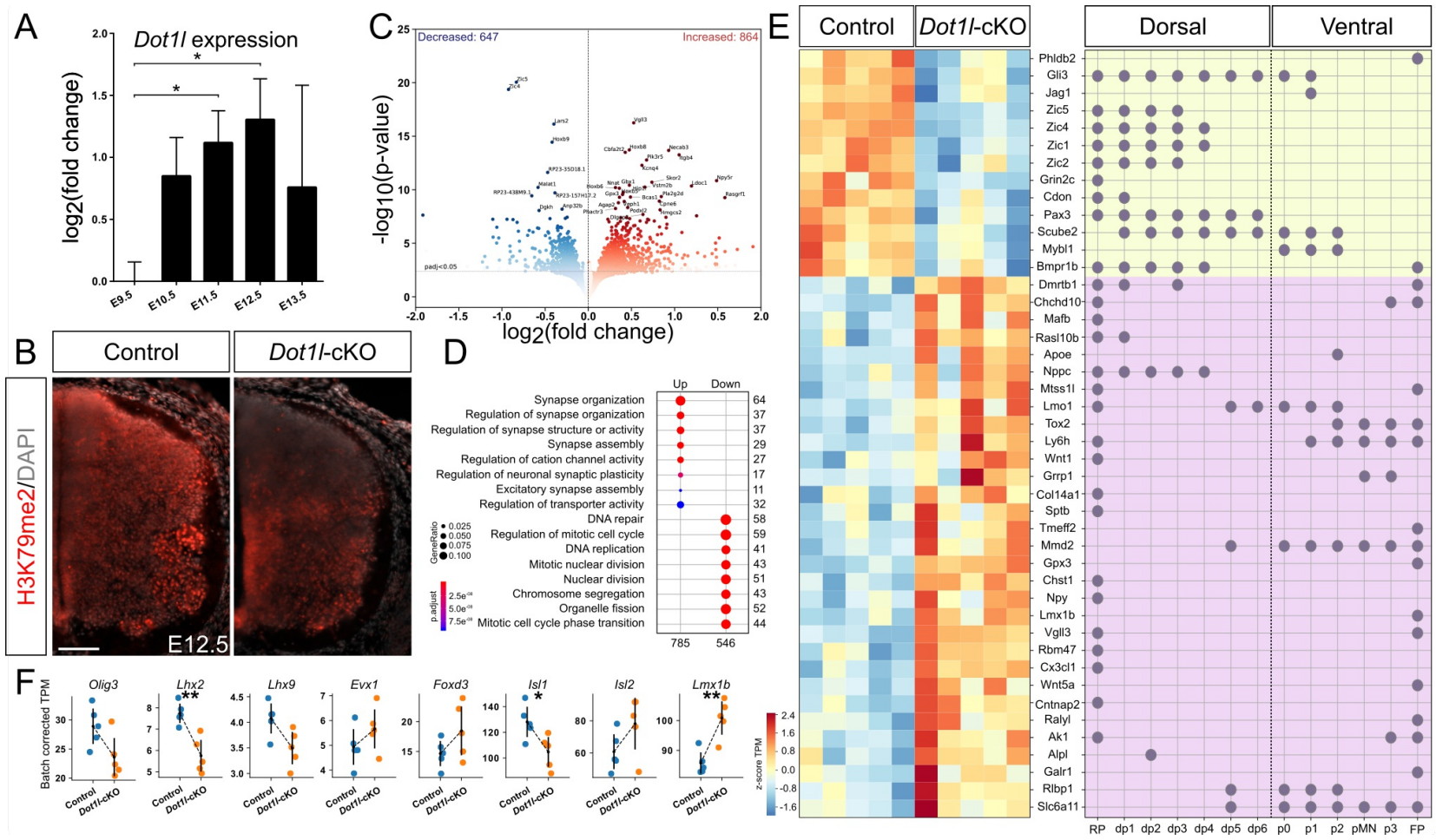

Figure 1

DOT1L activity during spinal cord neurogenesis supports progenitor maintenance and differentiation of dorsal interneurons. (A) qRTPCR analysis of Dot1l in wild type lumbar spinal cords at different embryonic stages (E9.5-E13.5) pooling of 3 individual embryos each, E12.5 and E13.5 n=8 from individual embryos). qRTPCR represented with mean \pm SEM. P-values were calculated with unpaired, two-tailed Student's t-test: $* p<0.05$ (B) Immunostaining H3K79me2 mark (red) and DAPI (gray) in E12.5 lumbar spinal cords in

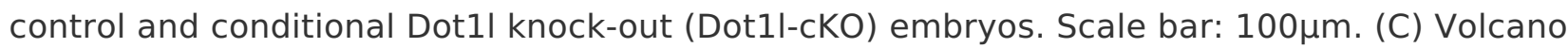
plot of differentially expressed genes (DEG) in Dot1l-cKO lumbar spinal cord compared to control littermates $(n=5)$ at E12.5. Colored, genes with increased (red) and decreased (blue) expression for adjusted $p<0.05$. (D) GO term analysis for biological processes of DEG in Dot1I-cKO. Scales of gene ratio and adjusted $p$-value reported to the bottom left side. Genes with upregulated biological processes in left column, with downregulated biological processes upon Dot1l-cKO in right column. Number of genes per term on the $\mathrm{Y}$ axis of the graph, total numbers up- or down-regulated on $\mathrm{X}$ axis. Threshold for enrichment analysis: 
adjusted $p<0.1$. ( $E$, left panel) Heatmap for DEGs in mutant littermates intersected with a gene list for expressed progenitor domain-specific genes extrapolated from (69). Colorcoding based on TPM z-score, scale at the bottom top left side. (E, right panel) 571 Annotation of progenitor domain-specificity relative to the genes intersected in the heatmap (69). (F) Scatterplot for DEGs with batch corrected TPM for interneuron markers (Olig3, Lhx2, Lhx9, Evx1, Foxd3, Isl1, Is|2, Lmx1b) in control (blue) and mutant (orange) samples, each dot corresponding to a single $\mathrm{n}$ in the transcriptome analysis. Error bars represent SD in the transcriptome. Adjusted $p$-values from the DEG are reported as $*$ adjusted $p<0.05, * *$ adjusted $\mathrm{p}<0.01$ 


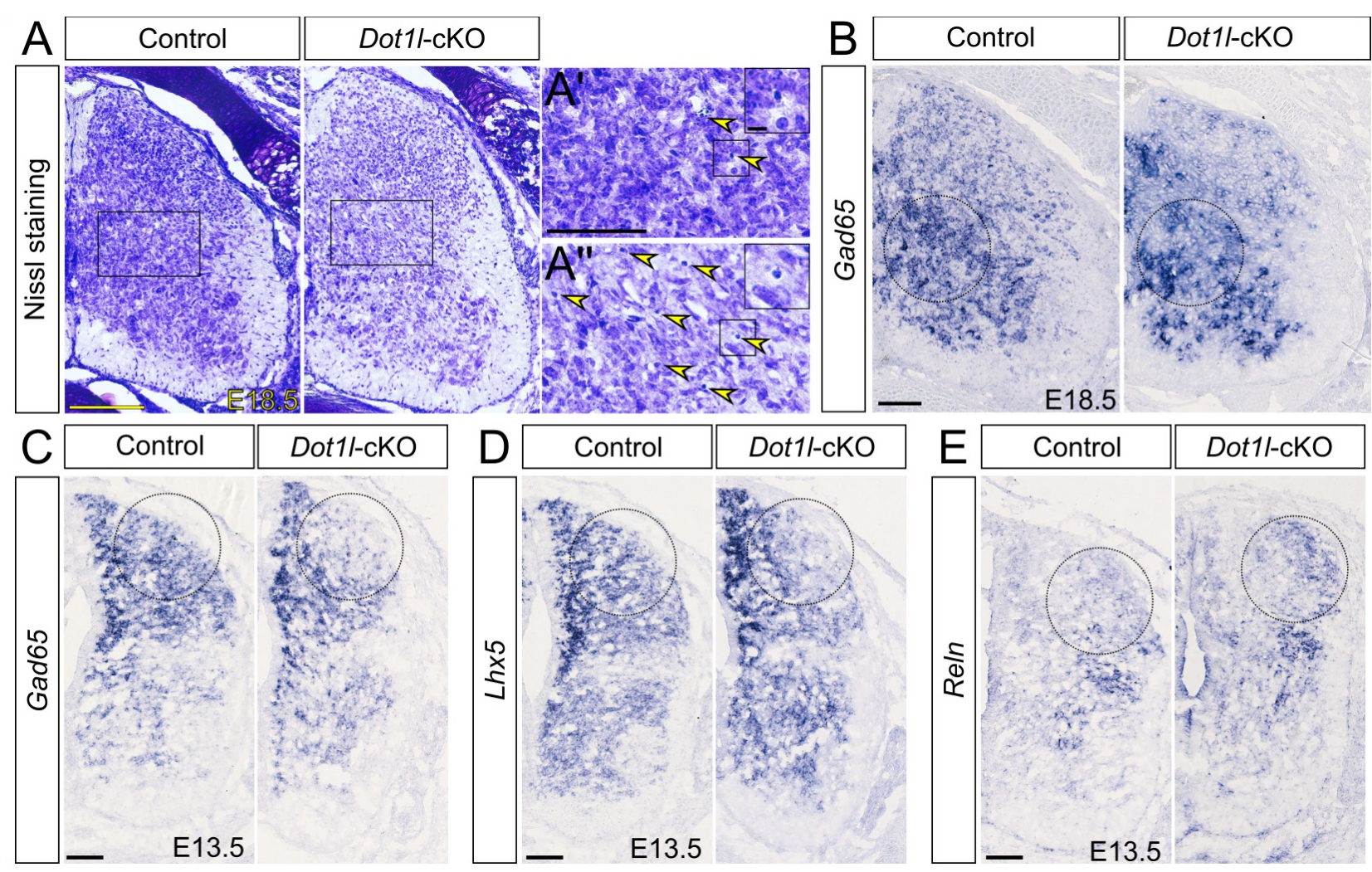

Figure 2

Dot1l-cKO causes loss of dorsal differentiation markers at E13.5 and extensive cell death at E18.5. (A) Nissl staining on prenatal (E18.5) lumbar spinal cord hemi-sections in control and Dot1l-cKO littermates. Black squares in the left panels refer to the higher magnifications to the right, $A^{\prime}$ for control and $A^{\prime \prime}$ for Dot1l-cKO. Within the right panels, boxed magnifications on top right refer to the smaller boxed areas showing pyknotic nuclei. Yellow arrowheads in panels to the right highlight pyknotic nuclei. Scale bar: overview $200 \mu \mathrm{m}$, magnification $\mathrm{A}^{\prime}$ and $A^{\prime \prime} 100 \mu \mathrm{m}$, blowup square $10 \mu \mathrm{m}$. (B, C) In situ hybridization (ISH) for Gad65 transcripts in lumbar spinal cord hemi-sections at E18.5 (B) and at E13.5 (C) in control and in Dot1l-cKO littermates. (D) ISH for Lhx5 transcripts in lumbar spinal cord hemi- sections at E13.5 of control and Dot1I-cKO. (E) ISH for Reln transcripts in lumbar spinal cord hemisections at E13.5 of control and Dot1I-cKO. Black circles: regions of interest described in the results. Scale bars: $100 \mu \mathrm{m}$. 

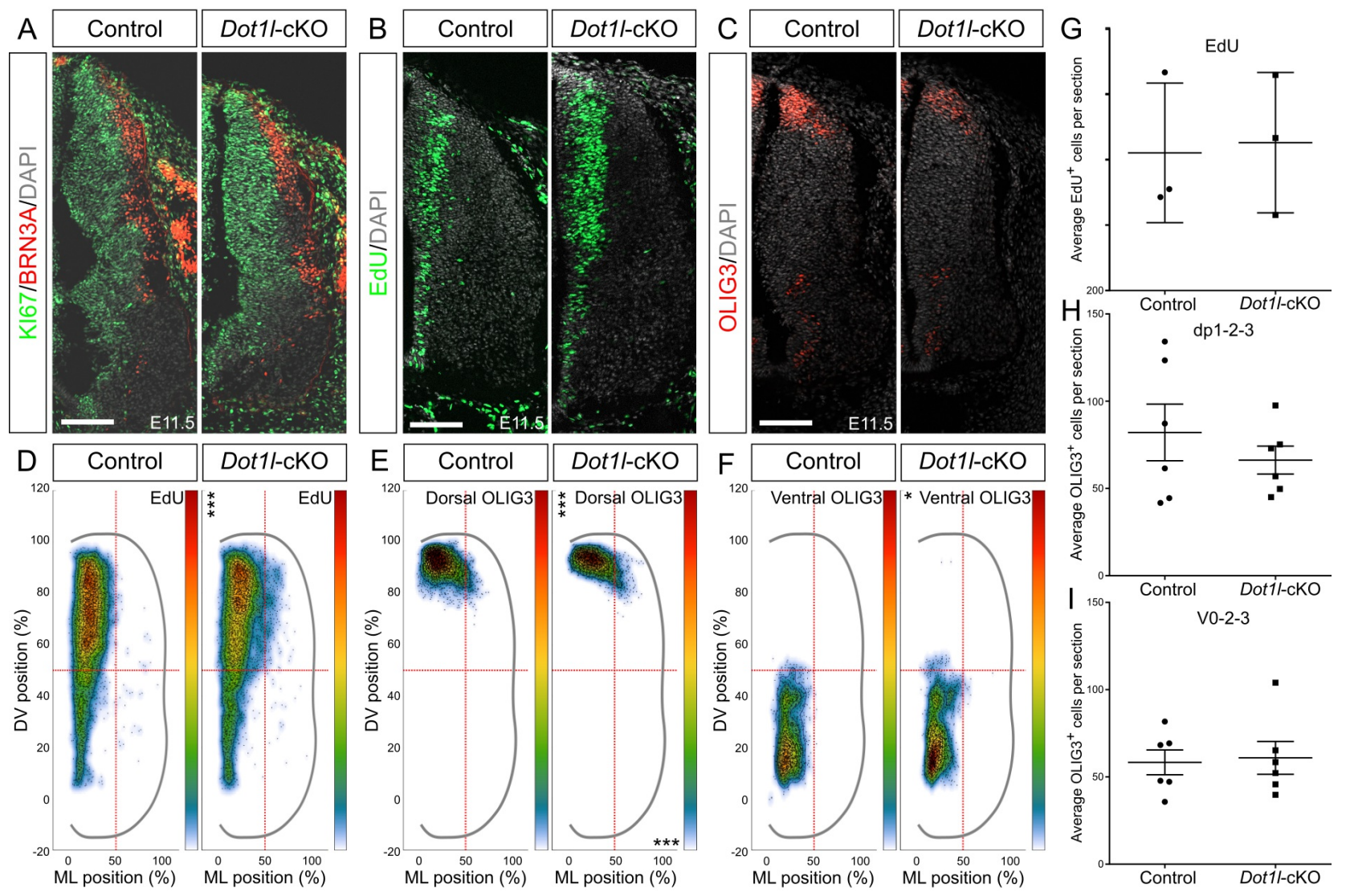

Figure 3

Dot1l-cKO results in defective early differentiation and migration of interneurons without affecting progenitor proliferation. (A) Immunostaining for KI67 (green), BRN3A (red) and DAPI (gray) on lumbar spinal cord hemi-sections at E11.5 from control and mutant littermates. (B) Representative images of immunostaining of EdU (green, 30 minutes pulse) and DAPI (gray) on E12.5 lumbar spinal cords in control and Dot1l-cKO embryos. (C) Representative images of immunostaining for OLIG3 (red) and DAPI (gray) on lumbar spinal cord hemi-sections at E11.5 from control and mutant littermates. Scale bars: $100 \mu \mathrm{m}$. (D, E, F) Density plots from EdU (D) and OLIG3 (E dorsal, F ventral interneuron). Density plot projections were analyzed by multivariate analysis for Hotelling's two-sample square test; * $\mathrm{p}<0.05,{ }^{* * *} \mathrm{p}<0.001$, stars 600 are reported on the $\mathrm{Y}$ axis (dorsoventral, DV) or $\mathrm{X}$ axis (mediolateral, ML) according to values on the 601 individual axes. (G, H, I) Quantitative analysis performed for EdU (G), dorsal OLIG3 interneurons (H) and 602 ventral OLIG3 interneurons (I). Quantifications represented with mean \pm SEM. P-values were calculated 603 
with unpaired, two-tailed Student's t-test. Per staining, 4 hemi-sections were counted for each n (EdU $604 \mathrm{n}=3$, OLIG3 n=6 both dorsal and ventral). Scale bars: $100 \mu \mathrm{m}$. 

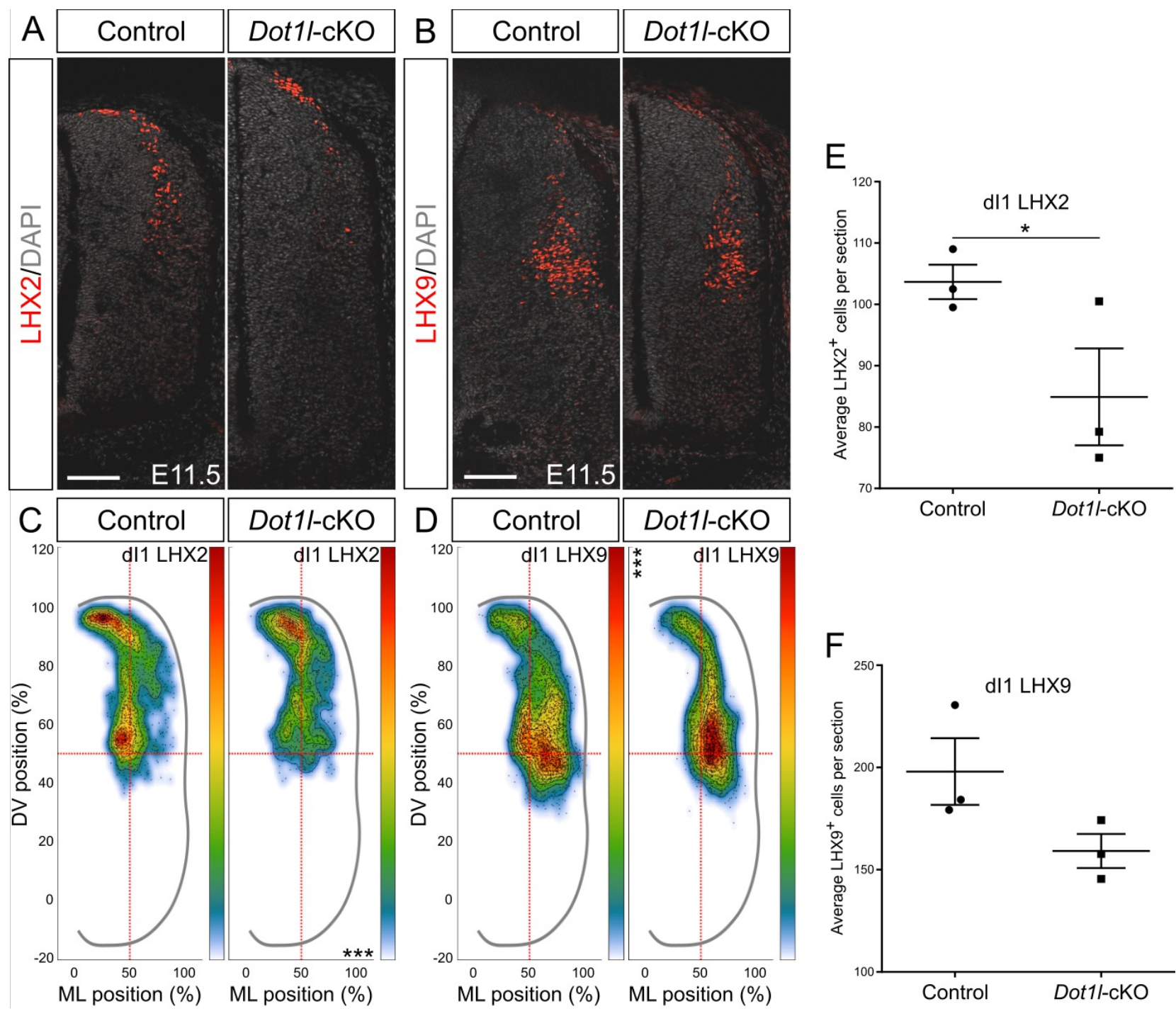

Figure 4

Dot1I-cKO decreases LHX2-dII interneurons and derails migration of the LHX9-dII interneurons at E11.5. (A, B) Representative immunostainings for LHX2 (red) and DAPI (gray) in (A) or LHX9 (red) and DAPI (gray) in (B) on spinal cord hemi-sections of control and mutant littermates at E11.5. Scale bars: $100 \mu \mathrm{m}$. (C, D) Density plots from LHX2 (C) and LHX9 (D). Density plot projections were analyzed by multivariate analysis for Hotelling's two-sample square test; ${ }^{* * *} \mathrm{p}<0.001$, stars are reported on the $\mathrm{Y}$ axis (dorsoventral, DV) or $\mathrm{X}$ axis (mediolateral, $\mathrm{ML}$ ) according to values on the individual axes. (E, F) Quantitative analyses of immunostainings for LHX2 (E) and LHX9 (F). Quantifications represented with mean \pm SEM. P-values were calculated with unpaired, one-tailed (E) or two-tailed (F) Student's t-test, $* p<0.05$. Per staining, 4 hemi-sections were counted for each $n(n=3)$. 

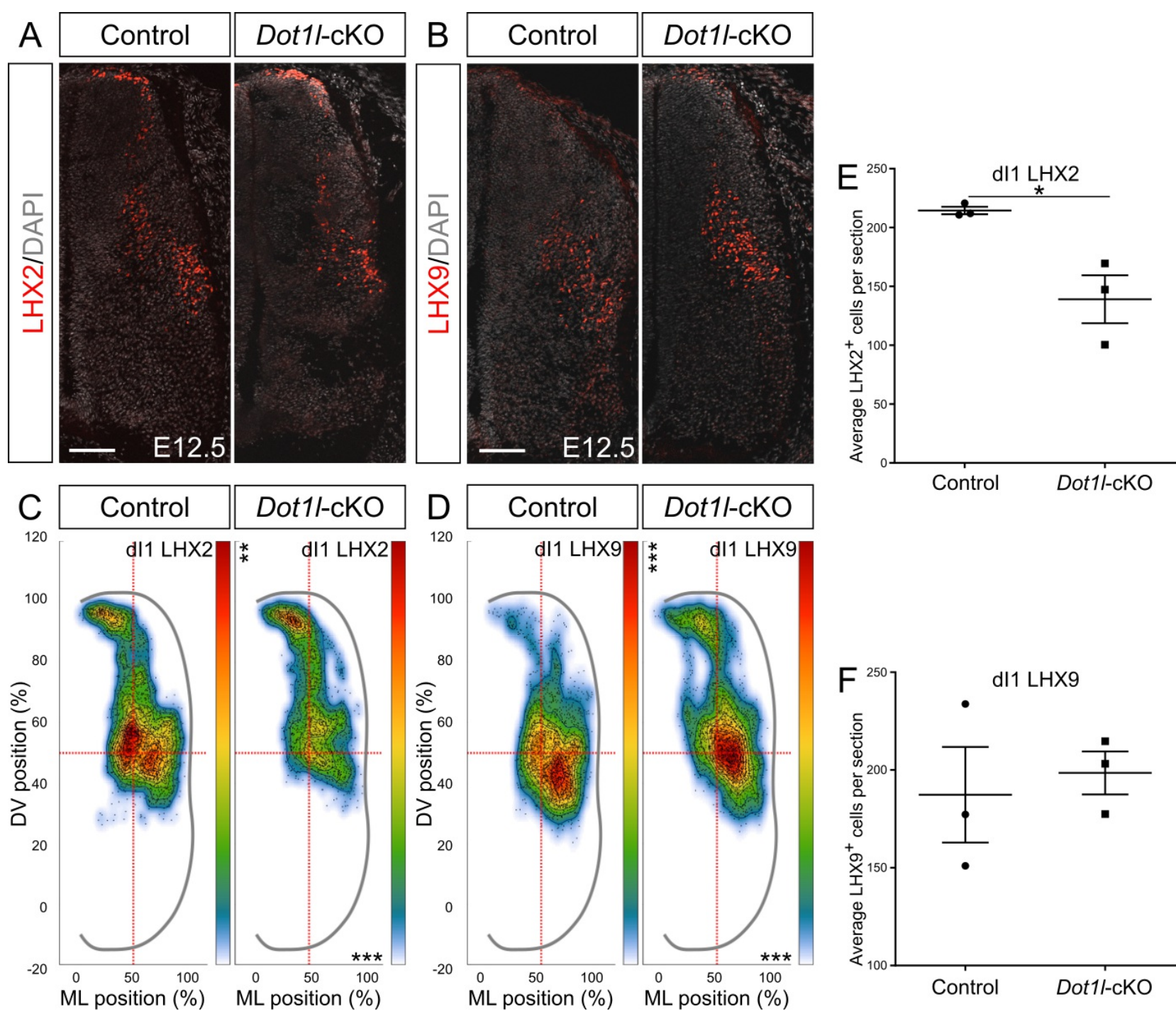

Figure 5

Dot1I-cKO decreases LHX2-dII interneurons and derails migration of the LHX9-dII

interneurons at E12.5. (A, B) Representative immunostainings for LHX2 (red) and DAPI (gray) in (A), and LHX9 (red) and DAPI (gray) in (B) on spinal cord hemi-sections of control and mutant littermates at E12.5. Scale bars: $100 \mu \mathrm{m}$. (C, D) Density plots from LHX2 (C) and LHX9 (D). Density plot projections were analyzed by multivariate analysis for Hotelling's two-sample square test; ${ }^{* * *} \mathrm{p}<0.001$, stars are reported on the $\mathrm{Y}$ axis (dorsoventral, DV) or $X$ axis (mediolateral, $M L$ ) according to values on the individual axes. $(E, F)$ Quantitative analyses of immunostainings for LHX2 (E) and LHX9 (F). Quantifications represented with mean \pm SEM. P-values were calculated with unpaired, one-tailed (E) or two-tailed (F) Student's t-test; $* p<0.05$. Per staining, 4 hemi-sections were counted for each $n(n=3)$. 

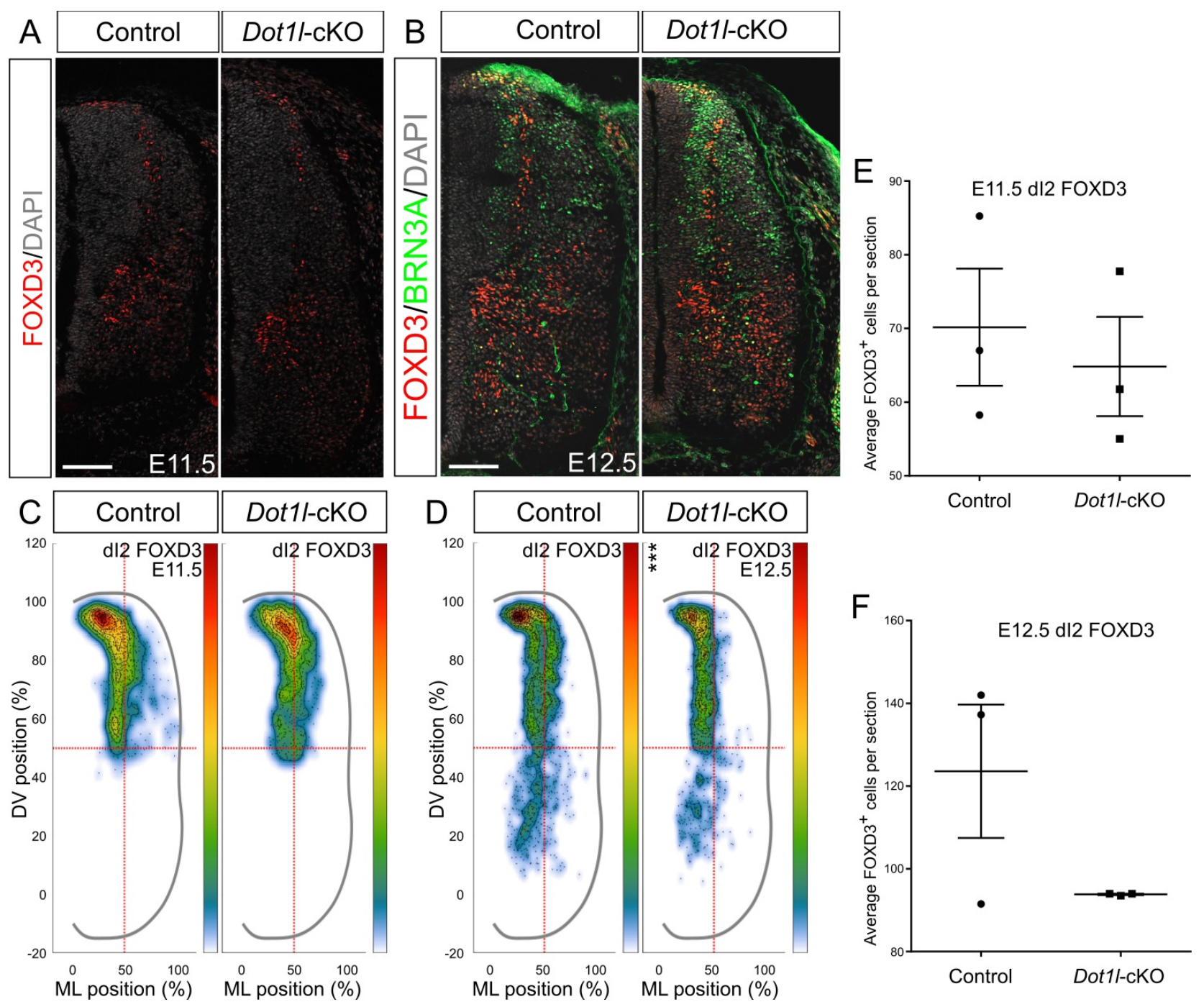

Figure 6

Dot1l-cKO impairs the dI2 migration path. (A, B) Representative immunostainings for dI2 and V1 interneurons at E11.5 (A) and E12.5 (B). In (A), dorsal FOXD3 (red) labels dI2 interneurons and ventral FOXD3 marks V1, while DAPI (gray) stains all the nuclei. In (B), dI2 are dorsally marked by FOXD3 (red) and ventrally by the costaining of FOXD3 and BRN3A (green). V1 in (B) are ventral cells labeled uniquely by FOXD3. Scale bars: $100 \mu \mathrm{m}$. (C, D) Density plots for dI2 interneurons at E11.5 (C) and E12.5 (D). Density plot projections were analyzed by multivariate analysis for Hotelling's two-sample square test; $* * * p<0.001$.

Stars are reported on the $\mathrm{Y}$ axis (dorsoventral, DV) or $\mathrm{X}$ axis (mediolateral, $\mathrm{ML}$ ) according to values on the individual axes. (E, F) Quantitative analyses of immunostainings for dI2 at E11.5 (E) and E12.5 (F). Quantifications represented with mean \pm SEM. P-values were 

counted for each $n(n=3)$.

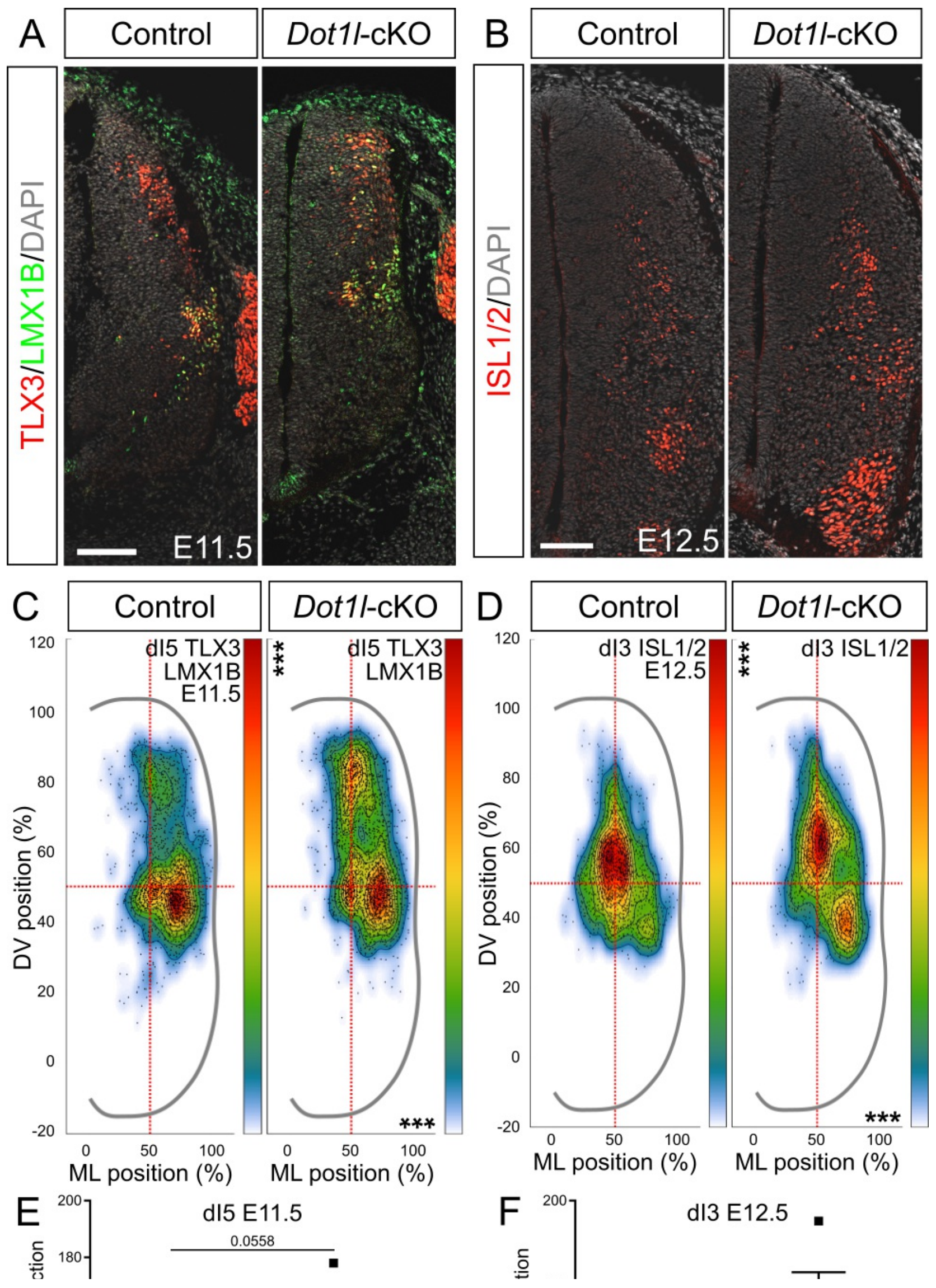



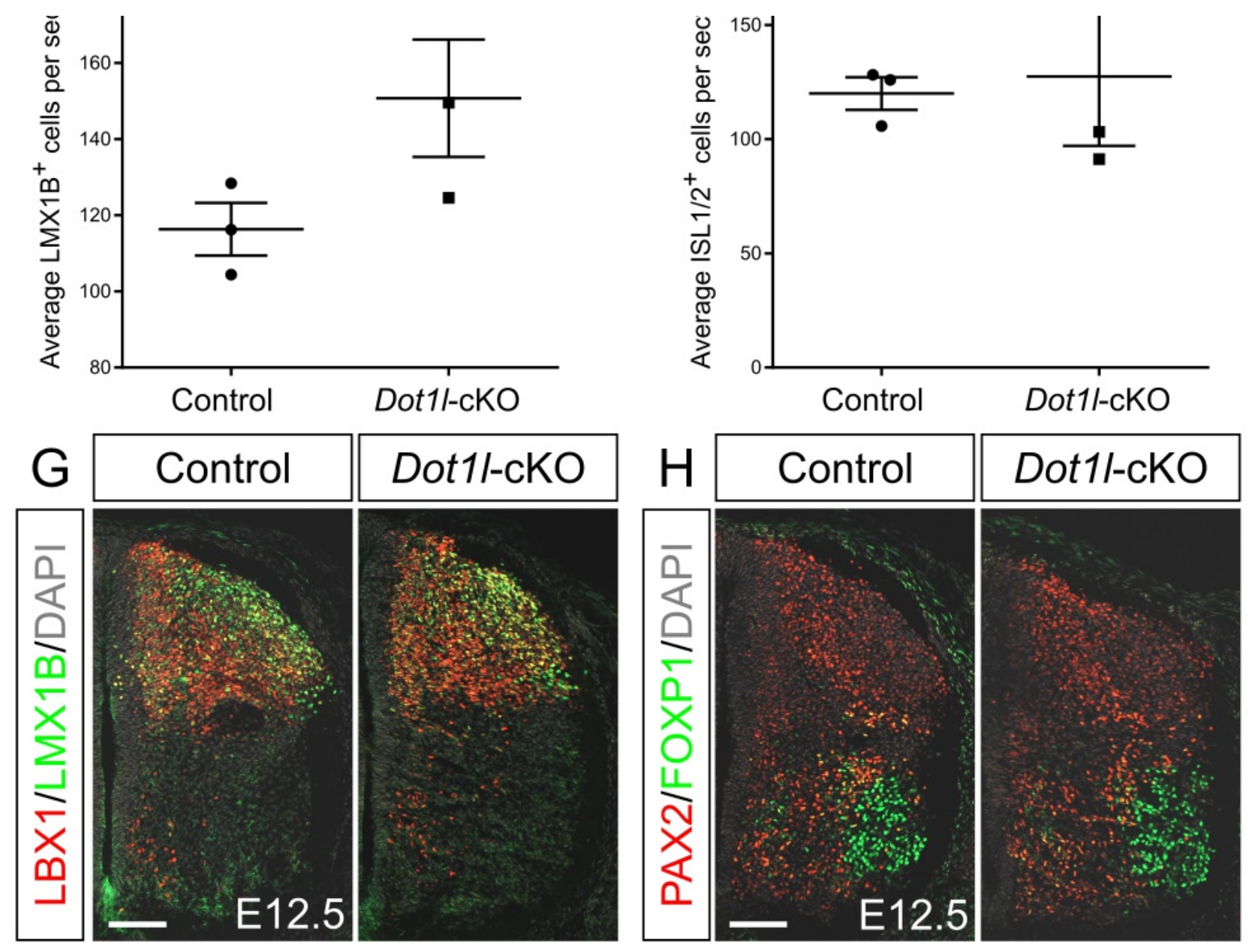

Figure 7

DOT1L differentially controls dI5 early positioning and dI3 late distribution. (A)

Representative immunostainings for $\mathrm{dl} 3$ and dl5 interneurons at E11.5. dI3 interneurons are labeled by TLX3 staining (red) and dI5 are labeled by both TLX3 and LMX1B (green), while DAPI (gray) stains all the nuclei. (B) Representative immunostainings for dI3 at E12.5. dl3 interneurons are labeled by ISL1/2 staining (red), while DAPI (gray) counterstains all the nuclei. (C, D) Density plots for dI5 interneurons at E11.5 (C) and dI3 interneurons at E12.5 (D). Density plot projections were analyzed by multivariate analysis for Hotelling's twosample square test; $\dagger p=0.08, * * * p<0.001$. Stars are reported on the $Y$ axis (dorsoventral, $D V$ ) or $\mathrm{X}$ axis (mediolateral, $\mathrm{ML}$ ) according to values on the individual axes. $(\mathrm{E}, \mathrm{F})$ Quantitative analyses of immunostainings for dl5 at E11.5 (E) and dI3 at E12.5 (F). (G, H) Representative immunostainings of LBX1 (red) and LMX1B (green) for excitatory interneurons (G) and PAX2 (red) in costaining with FOXP1 (green) for inhibitory interneurons 
(H) at E12.5. Scale bars: $100 \mu \mathrm{m}$. Quantifications represented with mean \pm SEM. P-values were calculated with unpaired, two-tailed Student's t-test. Per staining, 4 hemi-sections were counted for each $n(n=3)$.
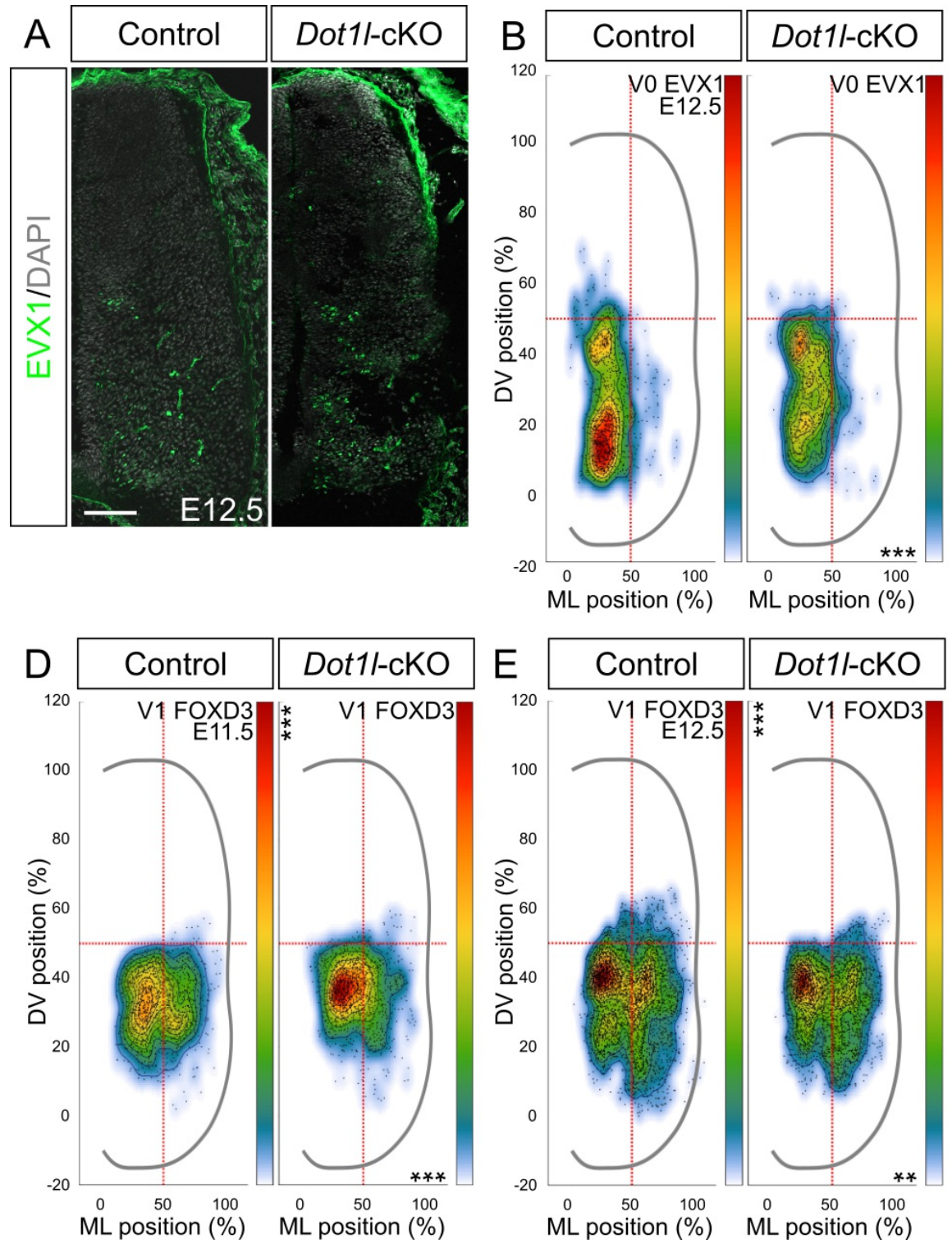

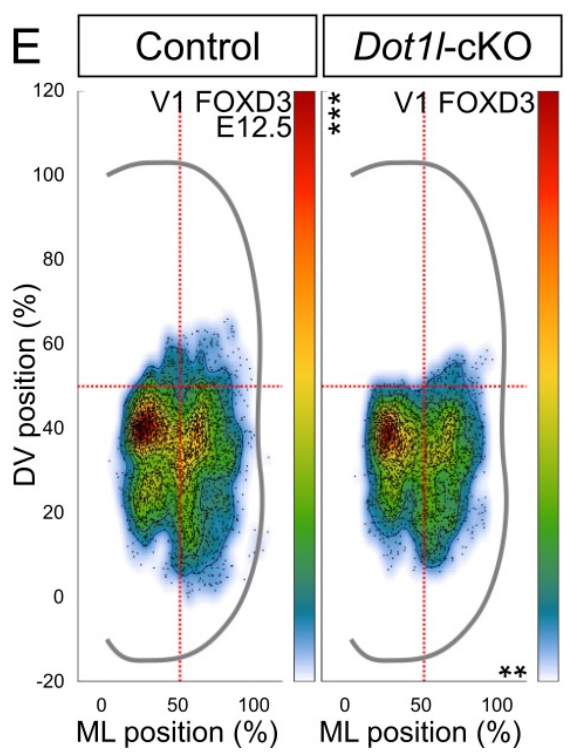

Figure 8

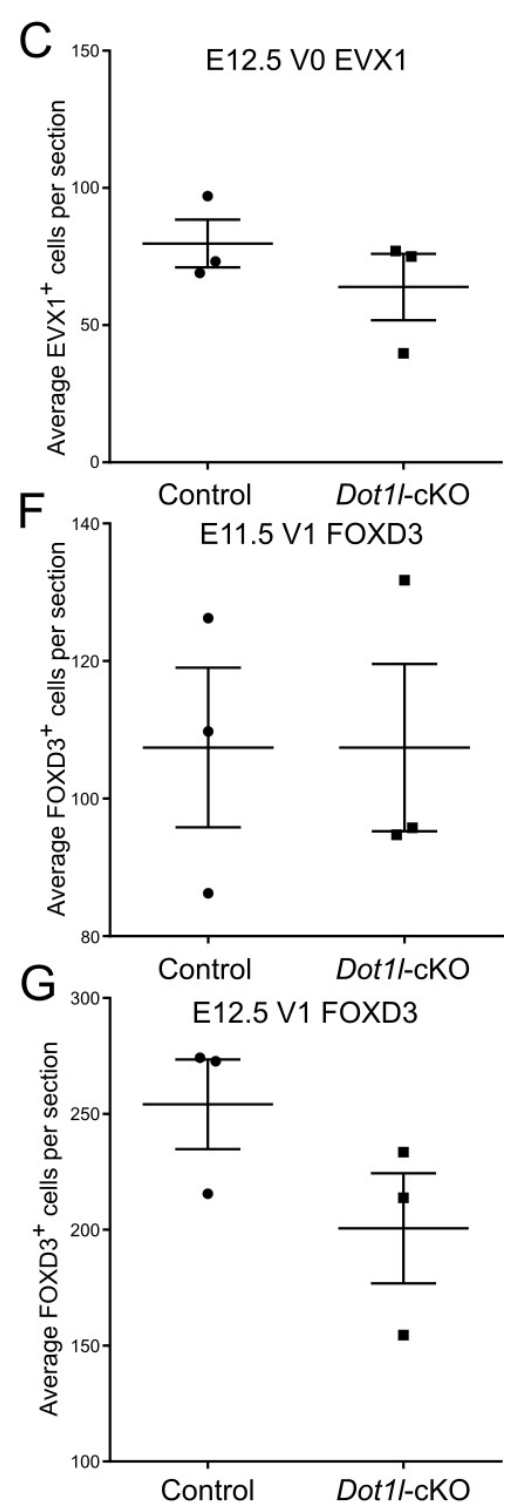

DOT1L depletion at E12.5 causes distributional defects of $\mathrm{V} 0$ and reduces $\mathrm{V} 1$ migration. $(\mathrm{A})$

Representative immunostainings for EVX1 (green) and DAPI (gray) on spinal cord hemisections of control and mutant littermates at E12.5. Scale bars: $100 \mu \mathrm{m}$. (B) Density plots from EVX1-expressing cells. (C) Quantitative analysis of EVX1, represented with mean \pm SEM. (D, E) Density plots for V1 interneurons from Figure 6A (E11.5, ventral cells FOXD3expressing) and 6B (E12.5, ventral cells FOXD3 single-labeled), respectively at E11.5 (D) 
and E12.5 (E). Density plot projections were analyzed by multivariate analysis for Hotelling 's two-sample square test; ** $\mathrm{p}<0.005, * * * \mathrm{p}<0.001$, stars are reported on the $\mathrm{Y}$ axis (dorsoventral, DV) or X axis (mediolateral, ML) according to values on the individual axes. $(F, G)$ Quantitative analyses of immunostainings for FOXD3-positive V1 at E11.5 (F) and E12.5 (G). P-values were calculated with unpaired, two-tailed Student's t-test. 4 hemisections were counted for each $n(n=3)$.

\section{Supplementary Files}

This is a list of supplementary files associated with this preprint. Click to download.

figS3_GraydeCristoforis.png

figS5_GraydeCristoforis.png

figS2_GraydeCristoforis.png

figS4_GraydeCristoforis.png

figS1_GraydeCristoforis.png 